\title{
Low Voltage Light Switching of Hybrid-Type Cell Composed of (Polymer/Liquid Crystal) Composite System
}

\author{
Hwan-Kyeong Jeong, Hirotsugu KikuchI, and Tisato KaJIYAma ${ }^{\dagger}$ \\ Department of Chemical Science and Technology, Faculty of Engineering, \\ Kyushu University, 6-10-1, Hakozaki, Higashi-ku, Fukuoka 812-81, Japan
}

(Received August 27, 1996)

\begin{abstract}
Polymer/liquid crystal: LC) composite films consisting of a continuous LC phase embedded in a threedimensional network of polymer matrix were prepared by a solvent-induced phase separation method. The (composite film/LC/composite film) hybrid laminar cell was applied for a novel light switching device by using the (poly(diisopropyl fumarate): Pdi-iPF/LC) composite systems as the composite films. A new type of cell geometry was proposed to achieve a low voltage drive for the light scattering-light transmission switching. The distribution ratio of an applied external electric field in the composite film layer and the LC one was calculated based on the series-connected dielectric composite model. The magnitude of an imposed electric field to the composite film layer was larger than an applied external one in the case that the absolute magnitude of the complex dielectric constant of LC was larger than that of the composite film. A remarkably low voltage driving for the electro-optical switching with reasonably fast rise and decay response times was realized for the new hybrid cell.
\end{abstract}

KEY WORDS (Polymer/Liquid Crystal) Composite Film / Phase-Separated Structure / Hybrid Cell / Dielectric Properties / Electro-Optical Switching /

(Polymer/liquid crystal: LC) composite films were developed by one of the authors for the first time in $1979^{1,2}$ and recently, have been intensively studied as novel materials in light shutters, large-area displays, projection displays, switchable windows and other functional devices. $^{3-6}$ Two typical aggregation structures of the (polymer/LC) composite systems have been reported, that is, the bicontinuous LC(BCLC) or the self-supported $\mathrm{LC}(\mathrm{SSLC})^{7,8}$ and the nematic curvilinear aligned phase(NCAP) ${ }^{9}$ or the polymer dispersed LC(PDLC). ${ }^{10}$ The (polymer/LC) composite systems, in which continuous LC domains are embedded in a three dimensional network of matrix polymer, have attracted great attention since they show excellent mechanical properties, ease of fabrication, effective permselectivity toward gases and ions, ${ }^{1,11}$ and excellent electro-optical responses. ${ }^{8-10}$

The electro-optical switching of the (polymer/LC) composite film is based on electrically controlled light scattering. The light scattering of the composite film in an a.c. electric field -off state is basically caused by both mismatch in the refractive indices between the matrix polymer and liquid crystal, and spatial distortion of nematic directors. ${ }^{12,13}$ The magnitude of spacial distortion of nematic directors in the LC channels is strongly dependent on the aggregation structure of the component materials. Apparently, the aggregation structure of the composite film strongly depends on the components ratio and the solvent evaporation rate during the film preparation process. ${ }^{14,15}$ The composite films exhibit reversible light scattering-light transmission switching upon electric fields-off and -on states, respectively. Since the composite film does not need the polarizers, a bright display is expected. Other advantages of the composite films might arise from wide viewing angle, fast response and the absence of required surface treatment. On the other hand, there still remain some problems to be solved in the (polymer/LC) composite films, e.g.,

\footnotetext{
$\dagger$ To whom correspondence should be addressed.
}

weak back scattering, high driving voltage and a hysteresis in an applied electric voltage-light transmittance curve. $^{16,17}$

It was reported that when poly(diisopropyl fumarate) (Pdi-iPF) was used as the matrix polymer, little switching hysteresis was observed because of poor compatibility at the interface between polymer and LC. ${ }^{18}$ Although the Pdi-iPF possesses many superior characteristics as the matrix polymer for the composite film, such as little hysteresis, fast response speed, high contrast and excellent repeating stability, the driving voltage is fairly high.

In this study, the electro-optical properties of a novel type of hybrid cell consisting of the (Pdi-iPF/LC) composite films have been investigated. Also, the dielectric properties for the hybrid laminar cell have been discussed.

\section{EXPERIMENTAL}

\section{Materials}

The chemical structure and the physico-chemical properties of polymer and LC are given in Figure 1. Poly(diisopropyl fumarate) (Pdi-iPF) supplied by Nippon Oil \& Fats Co., Ltd. was used as the matrix polymer for the (polymer/LC) composite system. E8 was a nematic mixture several cyano-phenyl type LCs with a positive

$$
\text { Polymer }
$$

Poly(diisopropylfumarate)(Pdi-iPF)

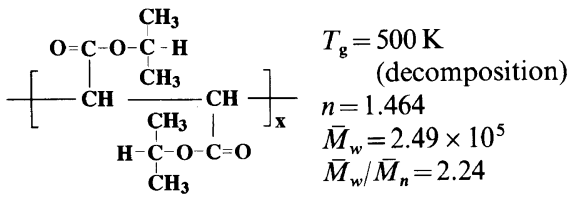

Liquid crystal

E8 (Nematic mixture)

$T_{\mathrm{KN}}=261 \mathrm{~K}, T_{\mathrm{NI}}=345 \mathrm{~K}, n_{\|}=1.774, n_{\perp}=1.527, \Delta n=0.247$

$\varepsilon_{\|}=21.3, \varepsilon_{\perp}=5.7, \Delta \varepsilon=15.6$

Figure 1. Chemical structure and physico-chemical properties of the matrix polymer and LC for the composite film. 
dielectric anisotropy.

\section{Sample Preparation and Investigation of Dielectric Prop- erties}

For the preparation of the (polymer/LC) composite films, the Pdi-iPF and E8 were dissolved in a common solvent such as a mixture of chloroform and toluene. The weight ratio of Pdi-iPF and E8 was 20/80 and the initial concentration of the solution was $5-10 \mathrm{wt} \%$. The solutions were spin-coated onto an indium tin oxide (ITO)-coated glass substrate. The ITO was a transparent electrode with a surface resistance of $10 \Omega$ /square. The film specimens were dried at room temperature in vacuo for $72 \mathrm{~h}$. The aggregation structure of the composite films was observed with a scanning electron microscope (SEM, Hitachi S-2150). For the SEM observation, the composite film was fractured in liquid nitrogen before the extraction of LC molecules with methanol at room temperature. The two types of LC cell were assembled as illustrated in Figure 2. The composite film of $6 \mu \mathrm{m}$ in thickness was sandwiched between two ITO-coated glass plates as shown in Figure 2(a). The dielectric properties were evaluated from the measurements of both capacitance and conductance for the composite film by using an impedance analyzer (Model 4192A, Yokogawa-HewlettPackard Ltd.). The electrode area and cell thickness were $400 \mathrm{~mm}^{2}$ and $6 \mu \mathrm{m}$, respectively. The hybrid laminar geometry consisting of the (composite film/LC layer/ composite film) as shown in Figure 2(b) was also prepared. The distance between ITO electrodes was fixed with Mylar films of $6 \mu \mathrm{m}$ thick.

\section{Electro-Optical Measurement}

In order to evaluate the electro-optical properties of the sample cell, light transmittance changes upon the application of an a.c. electric field were studied by the experimental set up reported elsewhere. ${ }^{19}$ The electrooptical properties were studied using a $\mathrm{He}-\mathrm{Ne}$ gas laser (wavelength: $632.8 \mathrm{~nm}$ ) as a light source. A rectangularwave with a frequency of $1 \mathrm{kHz}$ produced by a function generator was amplified by a power amplifier to drive the sample cell. A $\mathrm{He}-\mathrm{Ne}$ gas laser beam was passed through the sample cell perpendicular to the substrate and the transmitted light intensity through the sample cell without any polarizers was measured with a photodiode under the modulation of an applied a.c. electric field. The intensity of transmitted light was monitored with a digital storage oscilloscope (Kawasaki KDS-102) and was recorded with a personal computer. The distance between sample cell and photodiode was $305 \mathrm{~mm}$ and the sensing area of the photodiode was $6.6 \mathrm{~mm}^{2}$.

\section{RESULTS AND DISCUSSION}

\section{Morphology of (Polymer/LC) Composite Film}

In order to apply the composite film as a display device, a driving voltage lower than $10 \mathrm{~V}_{\text {rms }}$ is generally required because the driving method of light switching for the composite film must be carried out by the thin film transistor (TFT) driving mode. The phase-separated structure of the (polymer/LC) composite film is formed during the solvent evaporation from a uniform solution. It was reported that the phase-separated structure of the composite film was formed via spinodal decomposition and the LC domain size in the three-dimensional polymer network depended on the solvent evaporation rate. ${ }^{14,20}$ We have proposed that the phase-separated structure of the composite film was controlled by the solvent evaporation rate during the film preparation. Furthermore, the larger LC domain size was formed in the case of the slower solvent evaporation rate, and the electro-optical properties of the composite film is strongly affected by the LC domain size. For example, the driving voltage of light switching for the composite film decreased with an increase of the LC domain size. At present time, a high voltage over $40 \mathrm{~V}_{\mathrm{rms}}$ is necessary to drive the (Pdi$\mathrm{iPF} / \mathrm{E} 8$ : $40 / 60 \mathrm{wt} \%$ ) composite film of about $8 \mu \mathrm{m}$ in thickness. As shown in Figure 3, the (Pdi-iPF/E8) composite film exhibited a unique phase-separated structure that an entirely continuous E8 phase was embedded in a three-dimensional Pdi-iPF fibrillar network in the case of 20/80 (w/w\%) composition of Pdi-iPF and E8. Also, the sizes of LC domains in the composite film were not uniform, that is, they were randomly distributed in the polymer matrix. Therefore, it seems reasonable from the morphological feature that the (polymer/ LC) composite film can be treated as a binary dielectric composite composed of the two different dielectrics. The magnitude of an imposed electric field to the polymer and the LC phase being in a series connection are not always same as much as an external electric filed, because the magnitude of an a.c. electric field to each component is strongly dependent on the dielectric heterogeneous structure in the composite film. The dielectric heterogeneous aggregation structure can be easily controlled depending on the condition of the composite film preparation. In other words, the LC domain size and the distribution of LC domain size are very impor-

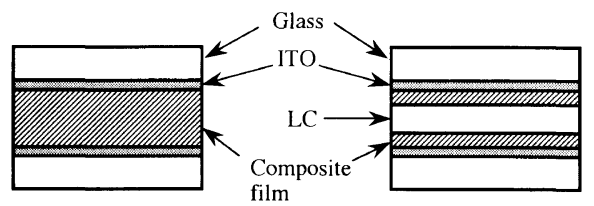

(a)

(b)

Figure 2. Schematic diagrams of the cell structure used for measuring the electro-optical properties of (a) the composite film, (b) the hybrid geometry of the (composite film/LC layer/composite film).

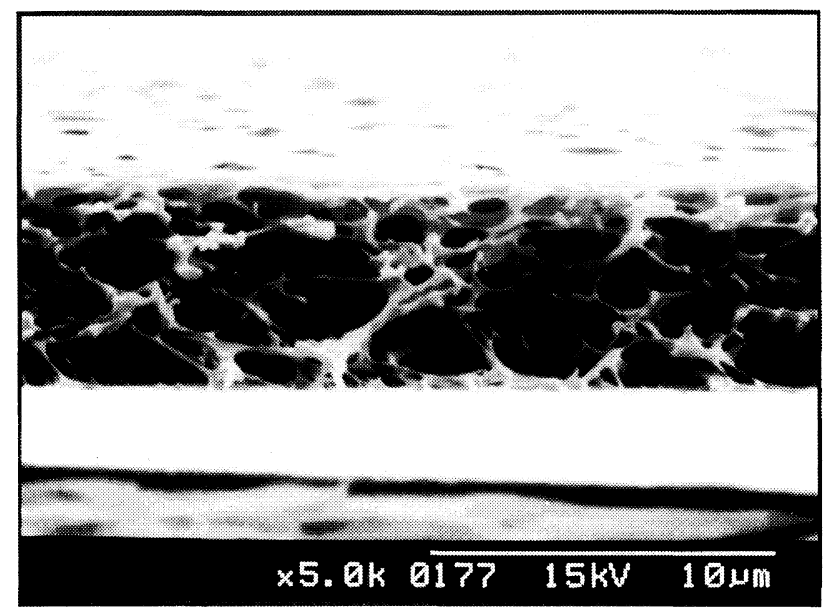

Figure 3. SEM photographs of the (Pdi-iPF/E8 $=20 / 80 \mathrm{wt} \%$ ) composite film. 


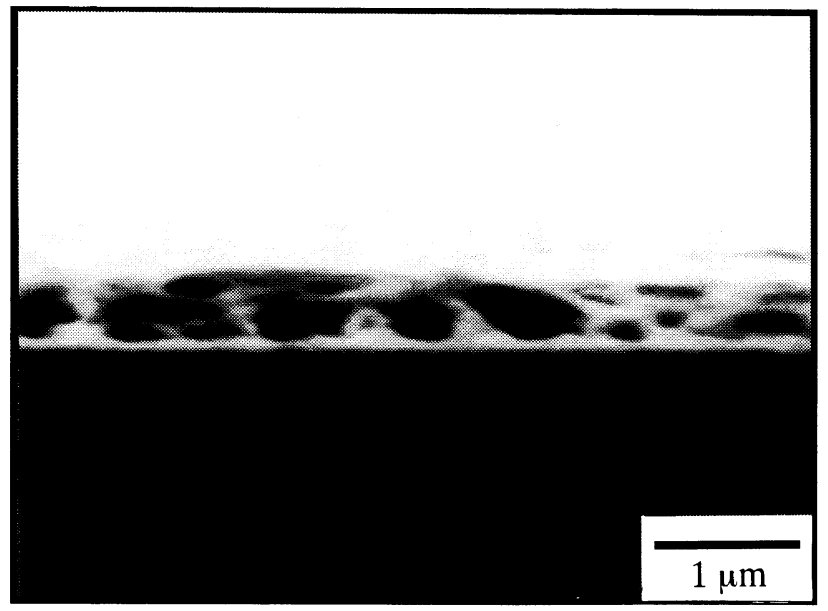

(a) Cross section

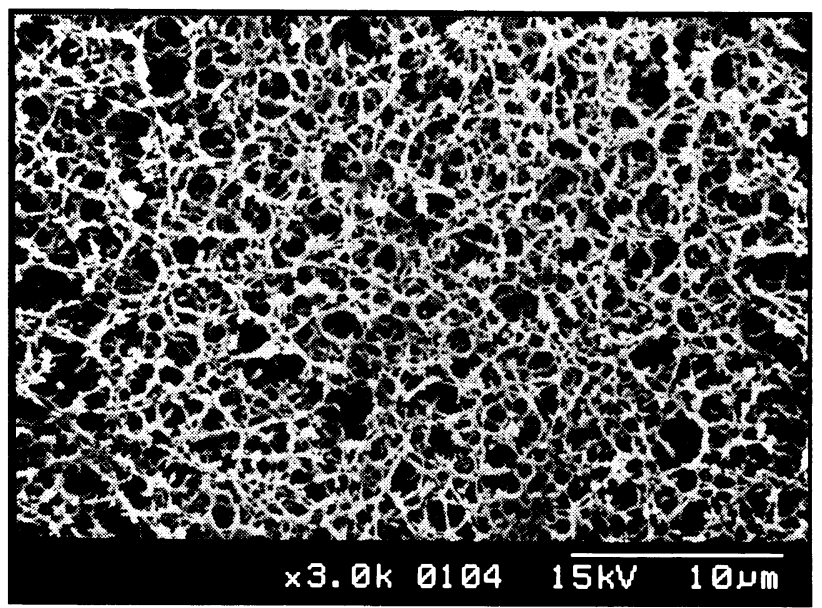

(b) Substrate facing surface

Figure 4. SEM photographs for the (Pdi-iPF/E8=20/80 wt $\%$ ) composite film of sub-micro meter in thickness. (a) cross section; (b) substrate facing surface.

tant factors to attain a low driving voltage of the composite film. Thus, in order to drive the electro-optical switching with a low driving voltage below $10 \mathrm{~V}_{\mathrm{rms}}$ for the composite film over $6 \mu \mathrm{m}$ in thickness, it is required to control not only the LC domain size but also its distribution. Unfortunately, there still remain uncontrollable problems in the composite film preparation, such as the LC domain dimension and its distribution. It is clear that the driving voltage of the (Pdi-iPF/E8) composite film generally decreases with an increase of E8 fraction and with a decrease of the film thickness. However, a desirable three-dimensional fibrillar network structure of the matrix polymer is not formed when the fraction of E8 increases more than $80 \mathrm{wt} \%{ }^{15}$ Also, an electric short-circuit should be considered, if the thickness of the composite film is thinner than a couple of $\mu \mathrm{m}$. Then, a new type of cell geometry, that is, the (composite film/LC/ composite film) layer cell shown in Figure 2(b) was proposed in this study to achieve a low voltage drive.

Figure 4 shows SEM photographs of the (Pdi-iPF/E8: $20 / 80 \mathrm{wt} \%$ ) composite film of sub- $\mu \mathrm{m}$ order in thickness after the extraction of E8 with methanol. The composite film exhibited a unique phase-separated structure that an entirely continuous E8 phase was embedded in a three dimensional Pdi-iPF fibrillar network. Since the completely random network structure was formed even at the surface, it is reasonable to expect that the long range orientation of the $\mathrm{LC}$ molecules should be distorted not only in the composite film but also in the vicinity of the surface of the composite film, as schematically shown in Figure 5(a). Therefore, a strong light scattering should be occurred for the hybrid cell in the absence of an electric field, mainly due to both the spatial distortion of LC directors and refractive index mismatching between polymer and LC as shown in Figure 5(a). On the other hand, since the LC molecules were aligned along the electric field upon the application of an electric field in the direction perpendicular to the substrate surface, the hybrid cell turned into transparent as shown in Figure 5(b). Then, a reversible turbid-transparent switching could be performed upon electric fields-off and on, respectively.

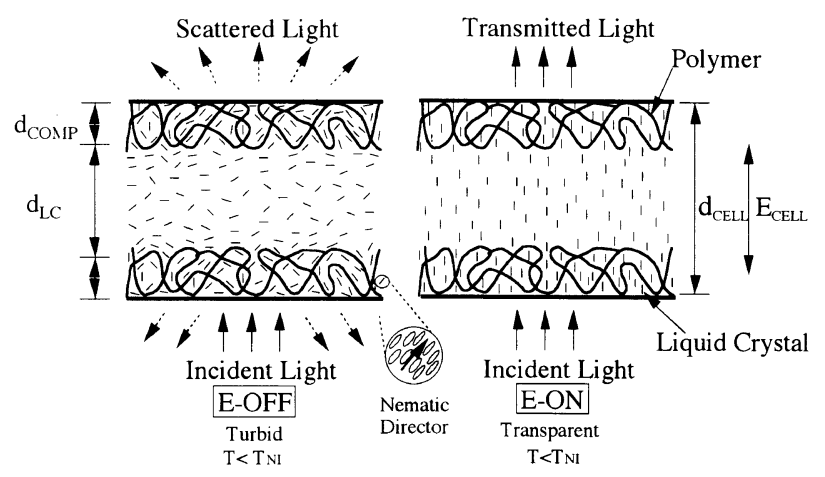

(a)

(b)

Figure 5. Schematic representation of the turbid and transparent states for the hybrid cell consisting of the composite film.

\section{Dielectric Analysis of the Hybrid Cell Consisting of} (Polymer/LC) Composite Film

The magnitudes of an effective applied electric field to the composite film layer and the LC one are not always the same, because of the difference in the absolute value of complex dielectric constants of the composite film and the LC layer sandwiched between composite films. Here, let us consider the magnitudes of an electric field distributed to the composite film layer and the LC one toward that an external field, based on the series-connected dielectric composite model. ${ }^{21,22}$ When an alternating electric field, $E$, is applied to a dielectric body, a complex electric displacement, $D^{*}$, is given by eq 1

$$
D^{*}=\varepsilon^{*} E
$$

where $\varepsilon^{*}$ is the complex dielectric constant expressed by eq 2 and the absolute value of $\varepsilon^{*}$ is written by using a dielectric constant, $\varepsilon^{\prime}$, and a dielectric loss factor, $\varepsilon^{\prime \prime}$, as given by eq 3 .

$$
\begin{gathered}
\varepsilon^{*}=\varepsilon^{\prime}-i \varepsilon^{\prime \prime} \\
\left|\varepsilon^{*}\right|=\left[\varepsilon^{\prime 2}+\varepsilon^{\prime \prime 2}\right]^{1 / 2}
\end{gathered}
$$

Since the composite film layer and the LC one are connected in series, the magnitude of $D^{*}$ along in the direction perpendicular to the film surface is constant in 
each layer as expressed by eq 4 and an electric field applied to the cell, $E_{\mathrm{CELL}}$, is given by eq 5 .

$$
\begin{gathered}
\left|D^{*}\right|=\left|\varepsilon_{\mathrm{CELL}}^{*} E_{\mathrm{CELL}}\right|=\left|\varepsilon_{\mathrm{COMP}}^{*} E_{\mathrm{COMP}}\right|=\left|\varepsilon_{\mathrm{LC}}^{*} E_{\mathrm{LC}}\right| \\
E_{\mathrm{CELL}}=\phi_{\mathrm{COMP}} E_{\mathrm{COMP}}+\left(1-\phi_{\mathrm{COMP}}\right) E_{\mathrm{LC}}
\end{gathered}
$$

where $\phi_{\text {Comp }}$ is a ratio of the total thickness of the composite films to the thickness of the hybrid cell, $2 d_{\text {COMP }} / d_{\text {CELL }}$. The subscripts CELL, COMP, and LC represent the prepared cell, the composite film and the LC layer, respectively. From eq 4 , the ratio of the magnitudes of electric field applied to the composite film and the LC one is given by eq 6 .

$$
\frac{E_{\mathrm{COMP}}}{E_{\mathrm{LC}}}=\left|\frac{\varepsilon_{\mathrm{LC}}^{*}}{\varepsilon_{\mathrm{COMP}}^{*}}\right|
$$

Then, eq 7 can be derived from eq 5 and 6 .

$$
\frac{E_{\text {COMP }}}{E_{\text {CELL }}}=\left[\phi_{\text {СOMP }}+\left(1-\phi_{\text {СOMP }}\left|\frac{\varepsilon_{\text {COMP }}^{*}}{\varepsilon_{\text {LC }}^{*}}\right|\right]^{-1}\right.
$$

where

$$
\left|\frac{\varepsilon_{\mathrm{COMP}}^{*}}{\varepsilon_{\mathrm{LC}}^{*}}\right|=\left[\frac{\varepsilon_{\mathrm{COMP}}^{\prime 2}+\varepsilon_{\mathrm{COMP}}^{\prime \prime 2}}{\varepsilon_{\mathrm{LC}}^{\prime 2}+\varepsilon_{\mathrm{LC}}^{\prime \prime 2}}\right]^{1 / 2}
$$

The relative dielectric constant, $\varepsilon^{\prime} / \varepsilon_{0}$, and the relative dielectric loss factor, $\varepsilon^{\prime \prime} / \varepsilon_{0}$, were respectively evaluated from the measurements of both capacitance and conductance for the (Pdi-iPF/E8: 20/80 wt\%) composite film and E8. Here, $\varepsilon_{0}$ is the dielectric constant of vacuum. It was experimentally confirmed that the (Pdi-iPF/E8: $20 / 80 \mathrm{wt} \%$ ) composite film showed little change in the dielectric constant and had a very small dielectric loss factor in a frequency range above $1 \mathrm{kHz}$ studied here. In contrast, the dielectric constant and the dielectric loss factor for the composite film showed a considerable increase with a decrease in frequency in a low frequency range $(<1 \mathrm{kHz})$. This result might be due to the interfacial polarization between Pdi-iPF and E8. The magnitudes of $\varepsilon_{\text {СOMP }}^{\prime} / \varepsilon_{0}$ COMP and $\varepsilon_{\text {COMP }}^{\prime \prime} / \varepsilon_{0}$ COMP for the (Pdi$\mathrm{iPF} / \mathrm{E} 8: 20 / 80 \mathrm{wt} \%$ ) composite film were 6.5 and 0.6 at $1 \mathrm{kHz}$, respectively. Also, those for E8 were about 22.9 and 2.1 at $1 \mathrm{kHz}$, respectively. These experimental results clearly indicate that a ratio of the magnitude of the complex dielectric constants, $\varepsilon_{\text {COMP }}^{*} / \varepsilon_{\mathrm{LC}}^{*}$, is strongly dependent on the magnitude of $\varepsilon^{\prime}$ in each layer when the frequency of a driving electric field was about $1 \mathrm{kHz}$. Since the magnitudes of $\varepsilon^{\prime \prime} / \varepsilon_{0}$ for the composite film and the LC were very small in comparison with those of $\varepsilon^{\prime} / \varepsilon_{0}$, eq 7 can be reasonable replaced approximated by eq 9 .

$$
\frac{E_{\mathrm{COMP}}}{E_{\mathrm{CELL}}}=\left[\phi_{\text {СOMP }}+\left(1-\phi_{\text {СOMP }}\right)\left|\frac{\varepsilon_{\mathrm{COMP}}^{\prime}}{\varepsilon_{\mathrm{LC}}^{\prime}}\right|\right]^{-1}
$$

A driving electric field used in this study on the measurement of electro-optical switching was a rectangular wave of $1 \mathrm{kHz}$. Therefore, the magnitude of $E_{\text {Comp }} /$ $E_{\text {CELL }}$ given in eq 9 may be calculated to the magnitude of $\varepsilon_{\mathrm{COMP}}^{\prime} / \varepsilon_{\mathrm{LC}}^{\prime}$ as shown in Figure 6. The magnitude of $E_{\mathrm{COMP}} / E_{\mathrm{CELL}}$ increases with a decrease of the magnitude of $\varepsilon_{\mathrm{COMP}}^{\prime} / \varepsilon_{\mathrm{LC}}^{\prime}$. When the absolute value of $\varepsilon_{\text {COMP }}^{\prime} / \varepsilon_{\mathrm{LC}}^{\prime}$ is smaller than 1 , it turns out that $E_{\mathrm{COMP}}$ becomes larger than $E_{\mathrm{CELL}}$. Also, since E8 has a large positive dielectric anisotropy, the magnitude of $\varepsilon_{\mathrm{COMP}}^{\prime} / \varepsilon_{\mathrm{LC}}^{\prime}$ ap-

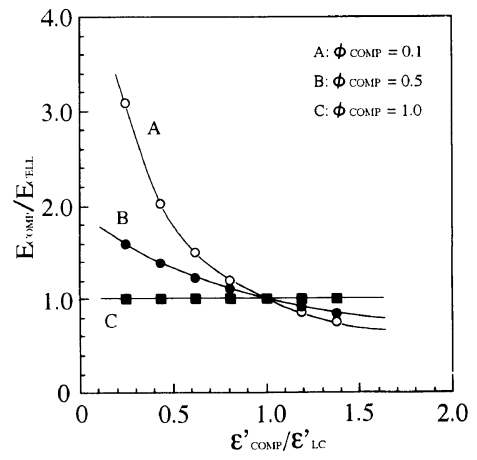

Figure 6. Magnitude of $\varepsilon_{\mathrm{COMP}}^{\prime} / \varepsilon_{\mathrm{LC}}^{\prime}$ and $\phi_{\mathrm{COMP}}$ dependence of the magnitude of $E_{\mathrm{COMP}} / E_{\mathrm{CELL}}$ for the hybrid cell.

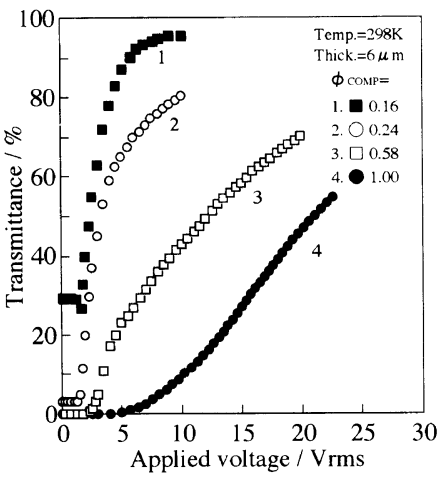

Figure 7. Applied voltage dependence of transmittance with the hybrid cells $(1-3)$ and the conventional composite cell (4).

parently becomes much smaller due to an effective LC orientation upon the application of an electric field. This clearly indicates that an electric field imposed to the composite film layer becomes larger than the externally applied one when the absolute value of $\varepsilon_{\mathrm{COMP}}^{\prime} / \varepsilon_{\mathrm{LC}}^{\prime}$ is smaller than 1. Furthermore, it is clear from Figure 6 that the magnitude of $E_{\mathrm{COMP}} / E_{\mathrm{CELL}}$ increases with a decrease in $\phi_{\text {Comp. In other words, the magnitude of }}$ $E_{\text {СомP }}$ should increase with a decrease in $\phi_{\text {сомp }}$. Therefore, it seems reasonable to consider that the driving voltage of the hybrid cell shown in Figure 2(b) or 5 should decrease compared with that of the conventional composite cell shown in Figure 2(a).

\section{Electro-Optical Switching of the Hybrid Cell}

Figure 7 shows the applied voltage dependence of the light transmittance for the hybrid cells $(1-3)$ and the conventional composite one (4). The driving voltage remarkably became lower and the transmittance change with an applied voltage became much steeper with decreasing $\phi_{\text {Сомр }}$. These trends agree fairly well with eq 9 . In the case of $\phi_{\text {Сом }}=0.16$, the electro-optical switching of the hybrid cell can be driven with a fairly low voltage below $10 \mathrm{~V}_{\mathrm{rms}}$ as shown in Figure 7. Since the magnitude of $\varepsilon_{\mathrm{COMP}}^{\prime} / \varepsilon_{\mathrm{LC}}^{\prime}$ was evaluated to be about 0.3 at $1 \mathrm{kHz}$, the magnitude of $E_{\mathrm{COMP}} / E_{\mathrm{CELL}}$ for the hybrid cell of $\phi_{\text {COMP }}=0.16$ was much larger than those of $\phi_{\text {COMP }}=0.24$ and $\phi_{\text {СоMP }}=0.58$. Therefore, it is reasonable to conclude that the decrease of the driving voltage for the hybrid cell with $\phi_{\text {COMP }}=0.16$ was strongly attributed to the higher magnitude of $E_{\text {Сом }}$ in comparison with that of an external electric field to the hybrid cell. 
Figure 8 shows the electric switching characteristics of the (Pdi-iPF/E8 $=20 / 80 \mathrm{wt} \%$ ) composite film under the different conditions of the composite film thickness and the driving voltage. The magnitudes of the rise response time, $\tau_{\mathbf{R}}$, and the decay response time, $\tau_{\mathrm{D}}$, were defined as the time period to change from $10 \%$ to $90 \%$ transmittance after the application of an a.c. electric field and that for the reverse transmittance change after turning off an a.c. electric field, respectively. The curve 1 corresponds to the switching curve driven with $40 \mathrm{~V}_{\mathrm{rms}}$ for the (Pdi-iPF/E8=20/80 wt\%) composite film. The excellent electro-optical characteristics were realized with the short response time below $10 \mathrm{~ms}$ and high contrast. However, in the case that the applied electric voltage was lower than $10 \mathrm{~V}_{\mathrm{rms}}$, an enough high contrast could not be obtained for the conventional composite film. In the case of the hybrid cell with $\phi_{\text {COMP }}=0.16$ and the driving voltage of $10 \mathrm{~V}_{\mathrm{rms}}$, though a short decay response time was achieved, the contrast was slightly poor and the magnitude of $\tau_{R}$ was fairly increased as shown in Figure 8. It is well known that $\tau_{R}$ and $\tau_{D}$ in the composite film are dependent on a number of different parameters, including the viscosity effect of LC, the driving voltage, and the anchoring effect at the interface between polymer and LC. ${ }^{16,17}$ The viscosity of E8 in the hybrid cell with $\phi_{\text {ComP }}=0.16$ should be similar to that of the conventional composite one with $\phi_{\text {COMP }}=1.0$, because Pdi-iPF and E8 are completely insoluble and are separated from each other. Namely, the viscosity effect of E8 might not be important to explain the response characteristics for the hybrid cell and the conventional composite film comprised of the same type of polymer and LC as this study. The anchoring effect on the LC molecular reorientation is oppositely exerted in the rise and the decay processes. That is, the anchoring effect acts as a resistance and a restoring force to the rise and the decay processes, respectively. Since the anchoring strength increases with a decrease of the size of LC domains, ${ }^{19}$ it is reasonable to consider that the net anchoring strength in the composite film is proportional to the area of the (polymer/LC) interface being dependent on the LC domain size. Then, the decrease of the LC domain size resulted in a long $\tau_{R}$ and a short $\tau_{D}$, respectively. Furthermore, the magnitude of $\tau_{\mathrm{D}}$ is not generally dependent on that of an electric field, because $\tau_{D}$ is measured after turning off an a.c. electric field. In contrast, the magnitude of $\tau_{\mathbf{R}}$ is strongly influenced by

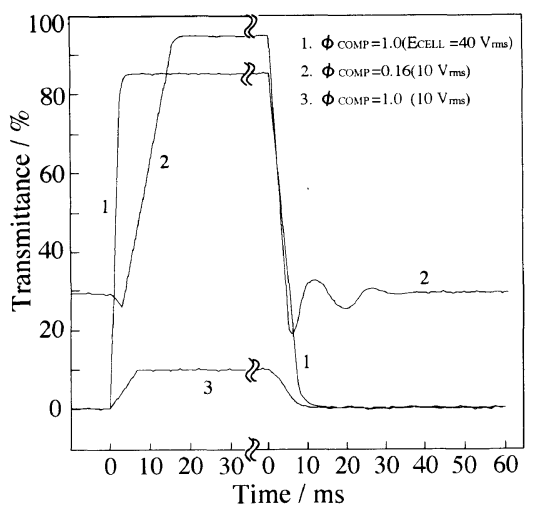

Figure 8. Electric switching characteristics of the hybrid cell and the conventional composite cell.

Polym. J., Vol. 29, No. 2, 1997 that of an electric field. That is, the magnitude of $\tau_{R}$ decreases with increasing the magnitude of an electric field. The magnitudes of $\tau_{R}$ and $\tau_{D}$ of the hybrid cell with $\phi_{\text {COMP }}=0.16$ driven by $10 \mathrm{~V}_{\mathrm{rms}}$ were $9.0 \mathrm{~ms}$ and $1.4 \mathrm{~ms}$, respectively, whereas, those of $\tau_{\mathbf{R}}$ and $\tau_{\mathbf{D}}$ of conventional composite film $\left(\phi_{\text {COMP }}=1.0\right)$ driven with $40 \mathrm{~V}_{\text {rms }}$ were $2.4 \mathrm{~ms}$ and $5.4 \mathrm{~ms}$, respectively. $\tau_{\mathrm{R}}$ for the hybrid cell of $\phi_{\mathrm{COMP}}=0.16$ was longer than that for the composite film with $\phi_{\text {COMP }}=1.0$, and $\tau_{\mathrm{D}}$ exhibited the opposite tendency for the cases of the hybrid cell and the composite film. Though the magnitudes of $\tau_{R}$ and $\tau_{D}$ cannot be explained on the basis of a quantitative analysis at present time, these tendencies on $\tau_{\mathrm{R}}$ and $\tau_{\mathrm{D}}$ might be caused by different anchoring strength due to the change of both the LC domain size and the driving voltage. These results indicate that the light scattering and transmission switching properties for the hybrid cells might be attributed to the composite film layers. As shown in Figures 3 and 4, the LC domain size of the composite film layer in the hybrid cell of $\phi_{\text {COMP }}=0.16$ was smaller compared with that of the composite film with $\phi_{\mathrm{COMP}}=$ 1.0. Then, the decrease of $\tau_{\mathrm{D}}$ in the hybrid cell with $\phi_{\text {COMP }}=0.16$ could be explained by the change in the LC domain size. In conclusion, the absolute value of $\varepsilon_{\text {COMP }}^{\prime} / \varepsilon_{\mathrm{LC}}^{\prime}$ from the experimental results on the measurement of dielectric properties was evaluated smaller than 1. Furthermore, it is clear from eq 9 that an electric field imposed to the composite film layer in the hybrid cell becomes larger than the externally applied one and the magnitude of $E_{\mathrm{COMP}}$ increases with a decrease in $\phi_{\text {сомp}}$. Therefore, it seems reasonable to conclude that the decrease of the driving voltage in the hybrid cell was generated by increasing $E_{\mathrm{COMP}}$ under the application of an electric field. Although there still remains some problems to be solved with respect to the contrast, a low voltage driving and the response time on electro-optical switching for the (Pdi-iPF/E8) composite film, the response characteristics have been successfully improved by the concept of the hybrid-type cell.

\section{CONCLUSIONS}

The (polymer/liquid crystal: LC) composite films exhibited a unique solvent-induced phase-separated structure that an entirely continuous LC phase was embedded in a three-dimensional Pdi-iPF fibrillarnetwork. A novel type of the (composite film/LC layer/composite film) hybrid cell was constructed especially to reduce the driving voltage. The analysis of the series-connected dielectric composite model theory indicated that the magnitude of $E_{\mathrm{COMP}} / E_{\mathrm{CELL}}$ in the hybrid cell was strongly dependent on the magnitudes of $\varepsilon_{\text {COMP }}^{*} / \varepsilon_{\mathrm{LC}}^{*}$ and $\phi_{\text {COMP }}$. It is clear from the model calculation that when the absolute value of $\varepsilon_{\text {COMP }}^{\prime} / \varepsilon_{\mathrm{LC}}^{\prime}$ is smaller than 1 , the magnitude of an electric field imposed to the composite film layer, $E_{\mathrm{COMP}}$, is larger than the externally applied one, $E_{\mathrm{CELL}}$, and also, the magnitude of $E_{\mathrm{COMP}} / E_{\mathrm{CELL}}$ increases with a decrease in $\phi_{\text {comp. }}$. A remarkably low voltage driving on the electro-optical switching, $10 \mathrm{~V}_{\mathrm{rms}}$, and the electric response characteristics being shorter than $10 \mathrm{~ms}$ was achieved for the hybrid cell with $\phi_{\text {COMP }}=0.16$. From the experimental results of dielectric properties, $\varepsilon_{\text {COMP }}^{\prime} / \varepsilon_{\mathbf{L C}}^{\prime}$ was evaluated about 0.3 . Therefore, it is reasonable to 
conclude that the magnitude of $E_{\mathrm{COMP}} / E_{\mathrm{CELL}}$ for the hybrid cell of $\phi_{\text {COMP }}=0.16$ was much larger than those of hybrid cells with $\phi_{\mathrm{COMP}}=0.24$ and $\phi_{\mathrm{COMP}}=0.58$ and the conventional composite film. The decrease of the driving voltage in the hybrid cell can be explained by increasing $E_{\text {COMP }}$ under the application of an electric field.

\section{REFERENCES}

1. T. Kajiyama, Y. Nagata, E. Maemura, and M. Takayanagi, Chem. Lett., 676 (1979).

2. T. Kajiyama, Y. Nagata, S. Washizu, and M. Takayanagi, J. Membrane Sci., 11, 39 (1982).

3. M. J. Sansone, G. Khanarian, T. M. Leslie, M. Stiller, J. Altman, and P. Elizondo, J. Appl. Phys., 67, 4253 (1990).

4. Y. Hirai, S. Niiyama, Y. Ooi, M. Kunigita, H. Kumai, M. Yuki, and T. Gunjima, SID 91 Digest, 594 (1991).

5. P. Van Konynenburg, R. Wipfler, and J. L. Smith, Proc. SPIE, 1080, 62 (1989).

6. K. Takizawa, H. Kikuchi, H. Fujikake, K. Kodama, and K. Kishi, J. Appl. Phys., 75, 3158 (1994).

7. T. Kajiyama, H. Kikuchi, M. Katayose, and S. Shinkai, New Polym. Mater., 1, 99 (1988).
8. T. Kajiyama, A. Miyamoto, H. Kikuchi, and Y. Morimura, Chem. Lett., 813 (1989).

9. J. L. Fergason, SID Int. Symp. Dig. Technol., 16, 85 (1985).

10. J. W. Doane, N. A. Vaz, B. G. Wu, and S. Zumer, Appl. Phys. Lett., 48, 269 (1986).

11. T. Kajiyama, A. Takahara, and H. Kikuchi, Polym. J., 23, 347 (1991).

12. A. Miyamoto, H. Kikuchi, Y. Morimura, and T. Kajiyama, New Polym. Mater., 2, 27 (1990).

13. H. Kikuchi, S. Moritomi, J. C. Hwang, and T. Kajiyama, Polym. Adv. Tech., 1, 297 (1990).

14. T. Kajiyama, K.-S. Park, F. Usui, H. Kikuchi, and A. Takahara, Proc. SPIE, 1911, 122 (1993).

15. K.-S. Park, H. Kikuchi, and T. Kajiyama, Polym. J., 26, 895 (1994).

16. H. Ono and N. Kawatsuki, Jpn. J. Appl. Phys., 33, 6268 (1994).

17. H. Kikuchi, J. Nishiwaki, and T. Kajiyama, Polym. J., 27, 1246 (1995).

18. T. Kajiyama, O. Yonekura, J. Nishiwaki, and H. Kikuchi, Proc. SPIE, 2175, 88 (1994).

19. H. Kikuchi, F. Usui, and T. Kajiyama, Polym. J., 28, 35 (1996).

20. C. Shen and T. Kyu, J. Chem. Phys., 102, 556 (1995).

21. H. Kikuchi, A. Miyamoto, S. Kobayasi, S. Morimura, and T. Kajiyama, J. Chem. Soc. Jpn., Chem. Ind. Chem., 434 (1990).

22. A. Miyamoto, H. Kikuchi, S. Kobayashi, Y. Morimura, and T. Kajiyama, Macromolecules, 24, 3915 (1991). 